\title{
Cutaneous Alternariasis in a Patient With Renal Transplant
}

\author{
Mustafa Demirci ${ }^{1}$; Nurten Baran ${ }^{1}$; Atilla Uzum ${ }^{2}$; Aylin Orgen Calli ${ }^{3}$; Sureyya Gul-Yurtsever ${ }^{1, *}$; \\ Tuna Demirdal ${ }^{4}$ \\ ${ }^{1}$ Department of Medical Microbiology, Ataturk Training and Research Hospital, Izmir Katip Celebi University, Izmir, Turkey \\ ${ }_{2}^{2}$ Department of Nephrology, Ataturk Training and Research Hospital, Izmir Katip Celebi University, Izmir, Turkey \\ ${ }^{3}$ Department of Pathology, Ataturk Training and Research Hospital, Izmir Katip Celebi University, Izmir, Turkey \\ ${ }^{4}$ Department of Infectious Diseases and Clinical Microbiology, Ataturk Training and Research Hospital, Izmir Katip Celebi University, Izmir, Turkey \\ ${ }^{*}$ Corresponding author: Sureyya Gul-Yurtsever, Department of Medical Microbiology, Ataturk Training and Research Hospital, Izmir Katip Celebi University, Izmir, Turkey. Tel: \\ +90-2322454545, E-mail: sgul71@yahoo.com \\ Received: March 21, 2014; Revised: November 11, 2014; Accepted: December 5, 2014
}

Introduction: Alternaria is a common saprophyte, which is usually not pathogenic in humans. Generally, local wounds infections of Alternaria occur with presence of immunosuppression factors such as HIV infection and renal transplant patients.

Case Presentation: We reported a case of wound infection induced by Alternaria spp. in a renal transplant patients. The main interest in this case was the rareness of the cutaneous alternariasis, its clinical aspects and good response to therapy. Recognition of Alternaria spp. as potential opportunistic pathogens is important for differential diagnosis of dermatological lesions, such as granulomatous or ulcerative lesions in immunocompromised patients.

Conclusions: Alternariasis or similar cases may be increased due to the increased number of immunosuppressed patients. From this point of view, skin lesions in these patients must be planned and microbiologically evaluated considering the molds.

Keywords: Cutaneous Alternariasis; Kidney Transplantation; Transplantation

\section{Introduction}

Alternaria spp. rarely causes disease in normal individuals despite its widespread distribution in nature. However, in the recent years, there is an increasing rate of infection caused by Alternaria spp. in immune-suppressed patients. The subspecies able to cause opportunistic infections in human are Alternaria alternata, A. tenuissima, A. infectoria and $A$. chartarum (1). The infections in human usually appear as skin or subcutaneous infections, but eye infections, invasive or noninvasive rhinosinusitis and onychomycosis could also be seen. The infection reveals itself as skin, subcutaneous infection or rhinosinusitis in immune-suppressed patients with risk factors like organ transplantation and Cushing syndrome. Onychomycosis is frequently associated with a history of nail trauma or contaminations with soil. Alternariasis responds well to classical antifungal treatment and skin, subcutaneous tissue and nail infections are well-treated with itraconazole $(2,3)$.

\section{Case Presentation}

A 32-year-old female patient with chronic renal failure due to secondary hypertensive nephrosclerosis and a history of renal transplantation from a cadaver 4 months ago after 7 years of peritoneal dialysis and 4 years of haemodialysis was reported. Most patients with genetic hemochromatosis have mutations of the HFE gene and excess amount of ferrum in blood. She had been receiving desferrioxamine treatment for the last year. The patient had been treated with antithymocyte globulin, tacrolimus, mycophenolate mofetil and corticosteroid for immune suppression and trimetoprim sulfamethoxazole and acyclovir for prophylaxis. The patient was currently receiving tacrolimus, mycophenolate mofetil and corticosteroid treatment. In the post transplantation period, a urinary infection was detected in patient and treated successfully with piperacillin and imipenem. After transplantation, the patient was discharged with a serum creatinine level of $0.7 \mathrm{mg} / \mathrm{dL}$. The patient arrived to our clinic with a complaint of ulcerative nodular lesion which is $2 \times 2 \mathrm{~cm}$ in size located at fronto-superior right-knee area (Figure 1).

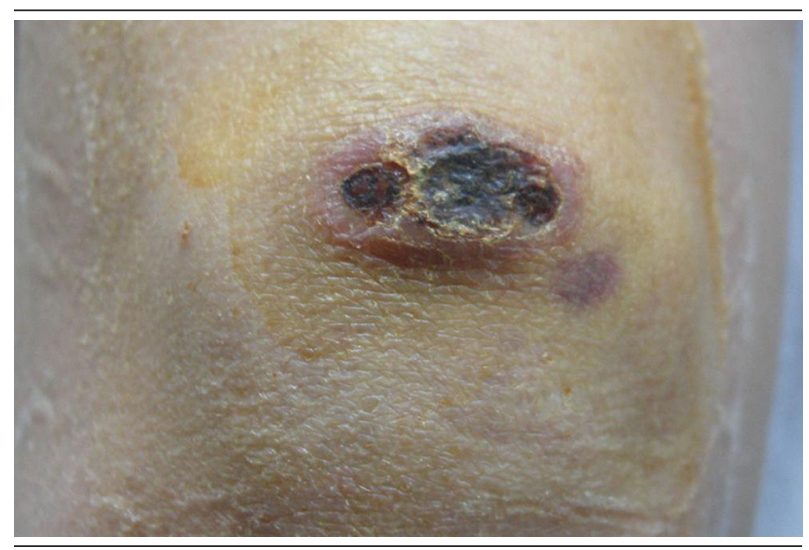

Figure 1. Skin Lesion in Elbow Area

Copyright (C) 2015, Ahvaz Jundishapur University of Medical Sciences. This is an open-access article distributed under the terms of the Creative Commons Attribution-NonCommercial 4.0 International License (http://creativecommons.org/licenses/by-nc/4.0/) which permits copy and redistribute the material just in noncommercial usages, provided the original work is properly cited. 


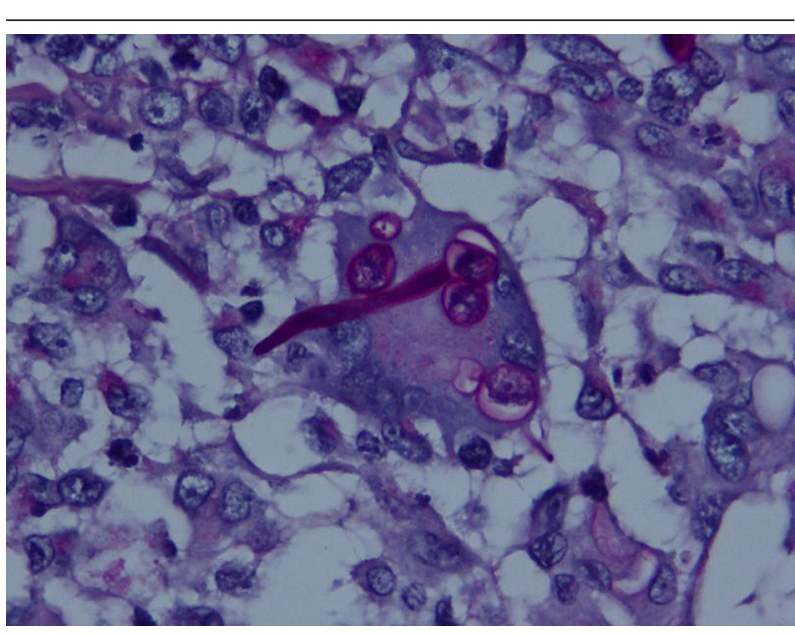

Figure 2. Fungal Elements Seen in Pathologic Examination of Biopsy

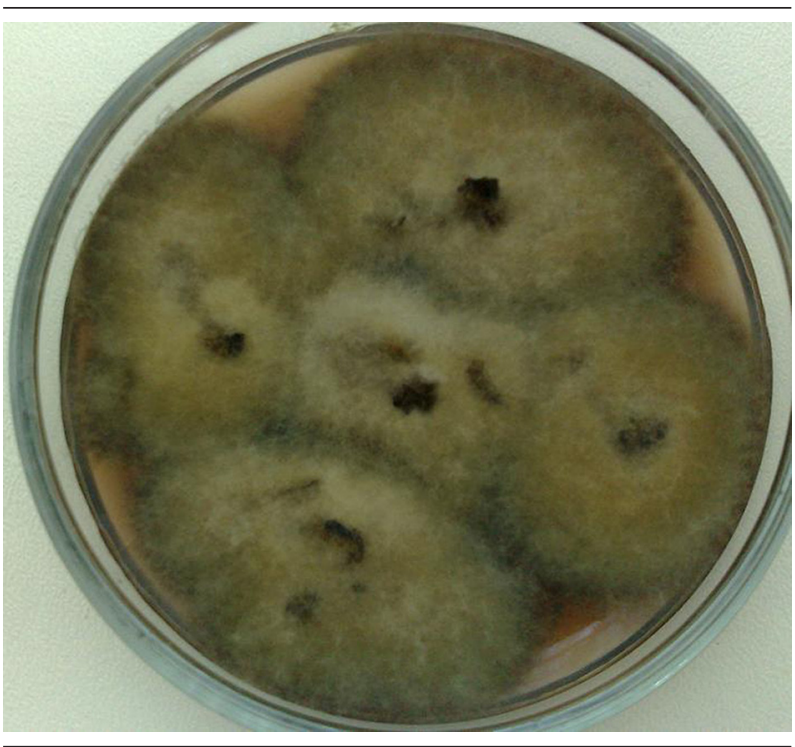

Figure 3. Appearance of Alternaria spp. Colonies Growth in Microbiological Examination

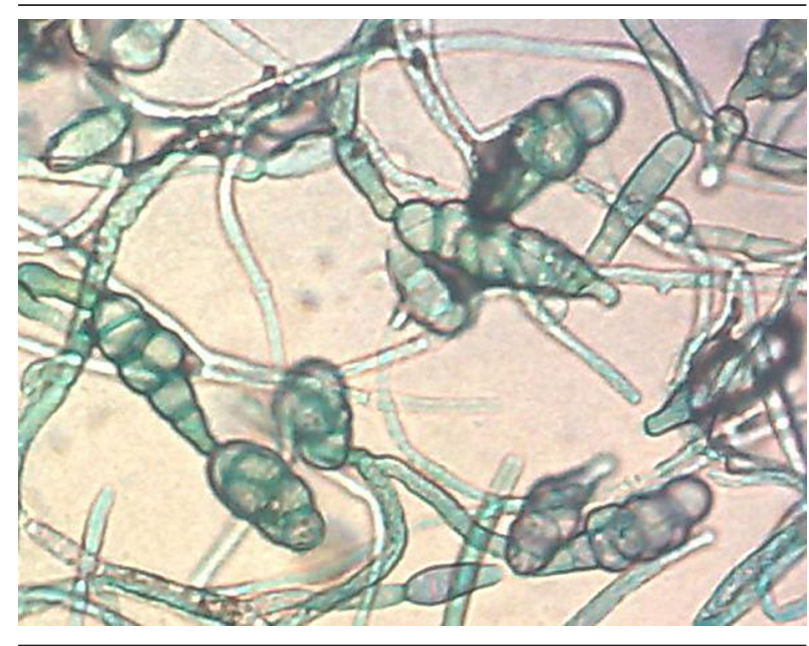

Figure 4. Alternaria spp. Macroconidia Isolated From Patients
The pathological examination of biopsy taken from the lateral border of ulcerative lesion revealed that it had pseudoepithelial hyperplasia, polymorph plasma cells, inflammation areas with histiocytes and hypha like constractions among inflammatory cells (Figure 2). Coagulase negative staphylococci were assessed in specimen cultures. In mycological Sabouraud dextrose agar (Himedia, India) cultures, greenish-brownish grey colonies with black punctations on the middle mold colonies with a loose structure grew within 3 - 4 days. The colonies evaluated to have fulfilling the whole plaque, browngreenish grey in color, black punctuations on the middle loose hypha structure (Figure 3). In micromorphological evaluation, hypha structure had compartments. At the distal ends of the structures, colorful macroconidia with dark brown colors, cylindrical, branching or breeding, horizontal or vertical comportments are detected (Figure 4). With these evaluations, the agent was thought to be $\mathrm{Al}$ ternaria spp. The patient received $200 \mathrm{mg} \times 2$ /day itraconazole as treatment together with reduction in mycophenolate mofetil intake to $360 \mathrm{mg} /$ day. Serum tacrolimus level inspected during antifungal treatment and reduced during itraconazole treatment. The macroscopic healing of the lesion was observed in the first two weeks and surgical nodulectomy performed in one month. The healing of lesion followed periodically and no recurrent or new lesion occurred.

\section{Discussion}

Although rare in normal individuals, Alternaria may be a cause of infection in immunosuppressed patients $(3,4)$. The port to enter the individual is usually the skin, which lost its uniform structure. Patients usually arrive with skin or subcutaneous infections. The infection may rarely appear as oculomycosis, sinusitis, onychomycosis and invasive infections $(4,5)$. In our case; there was no history of trauma, although the patient had skin infection. However, realizing the localization of skin lesions (elbows) implied that micro traumas had been the reason for skin infections. Cutaneous and subcutaneous Alternariasis cases may have visual clinical variations. In most patients, erythema, desquamation or red papule formation are inspected. Especially after corticosteroid treatments, erosions or ulcerations may develop. When the infection develops together with penetration caused by trauma, clinical view is like a purplish reddish plaque with an ulcer in the middle. Besides, in healthy patients, it can be seen as a crust ulcer form $(1,6)$. In our patient, nodular, crusted ulcerative form developed without trauma.

In our case, the molds grew in laboratory suggested Alternaria spp. due to colonial structure, hypha structure, localization of macroconidia on hyphae, horizontal or vertical compartments and tendency of change in compartments structures due to hypha localization. In a study of 156 Alternariasis patients, the rate of skin infections was $88.4 \%$ and subcutaneous infections as 
$11.6 \%$ (1). Among these patients, $60 \%$ were reported in the four Mediterranean Countries (France, Italy, Spain and Greece) and the average age was 54 (Ranged 6 - 94); the prevalence in men was $65 \%$ and $35 \%$ in women. The reason for a higher prevalence in men was probably a higher rate of exposure to external effects $(1,5)$. Although ours is a Mediterranean country, a very limited number of Alternaria cases were reported and according to the literature, this was the fifth case reported in Turkey (7-10).

In a study of 128 patients, predisposing factors of Alternariasis were 51 cases of transplantation (47 solid organ, 4 bone-marrow), 10 cases of Cushing Syndrome without receiving a transplantation and 67 cases of immunosuppressive treatment (1). Although the main cause of Alternariasis is A. alternata, (1), in a study of 156 cases, causative species were as follows; 59 (37.8\%) A. alternata and 23 case (14.7\%)A. tenuissima. Alternariasis or similar cases may be increased due to the increased number of immunosuppressed patients. From this point of view, skin lesions in these patients must be planned and microbiologically evaluated considering the molds.

\section{Authors' Contributions}

Study concept and design: Mustafa Demirci, Nurten Baran and Sureyya Gul-Yurtsever. Analysis and interpretation of data: Atilla Uzum, Aylin Orgen Calli and Tuna Demirdal. Drafting of the manuscript: Sureyya GulYurtsever. Critical revision of the manuscript for important intellectual content: Sureyya Gul-Yurtsever, Mustafa
Demirci, Nurten Baran, Atilla Uzum, Aylin Orgen Calli and Tuna Demirdal.

\section{References}

1. Pastor FJ, Guarro J. Alternaria infections: laboratory diagnosis and relevant clinical features. Clin Microbiol Infect. 2008;14(8):734-46.

2. Acland KM, Hay RJ, Groves R. Cutaneous infection with Alternaria alternata complicating immunosuppression: successful treatment with itraconazole. Br J Dermatol.1998;138(2):354-6.

3. Gurcan S, Piskin S, Kilic H, Temelli BA, Yalcin O. [Cutaneous infection caused by Alternaria alternata in an immunocompetent host]. Mikrobiyol Bul. 2009;43(1):163-7.

4. Gilaberte M, Bartralot R, Torres JM, Reus FS, Rodriguez V, Alomar A, et al. Cutaneous alternariosis in transplant recipients: clinicopathologic review of 9 cases. J Am Acad Dermatol. 2005;52(4):653-9.

5. Lespessailles E, Kerdraon R, Michenet P, Barthez JP, Mille C, Benhamou CL. Alternaria infection of the skin and joints. A report of two cases involving the hand. Rev Rhum Engl Ed. 1999; 66(10):509-11.

6. Ioannidou DJ, Stefanidou MP, Maraki SG, Panayiotides JG, Tosca AD. Cutaneous alternariosis in a patient with idiopathic pulmonary fibrosis. Int J Dermatol. 2000;39(4):293-5.

7. Akman A, Sakalli Cakcak D, Ozhak Baysan B, Yazisiz V, Terzioglu E, Ciftcioglu MA, et al. Cutaneous alternariosis in a patient with systemic lupus erythematosus. Lupus. 2007;16(12):993-6.

8. Baykal C, Kazancioglu R, Buyukbabani N, Celik AV, Yegenoglu Y, Kayacan SM, et al. Simultaneous cutaneous and ungual alternariosis in a renal transplant recipient. $\mathrm{Br} J$ Dermatol. 2000;143(4):910-2.

9. Yegenoglu, Y., Satana, D. , Erturan, Z. , Kiraz, M. , Uzun, M. , Ang, O. . Fungal Infections in Immunsupressed Patients. Turk Mikrobiyol Cem Derg. 2002;32:239-43.

10. Saray Y, Battaloglu, S., Demirbilek, M., Seckin, D. . Ungual alternariosis in a renal transplant recipient. Turkiye Klinikleri J Dermatol. 2005;15(3):160-3. 